\title{
Endovascular Repair of latrogenic Subclavian Artery Perforations Using the Hemobahn Stent-Graft
}

\author{
Antonio L. Bartorelli, MD; Daniela Trabattoni, MD; \\ Marco Agrifoglio, MD; Stefano Galli, MD; Luca Grancini, MD; and \\ Rita Spirito, MD
}
From the Centro Cardiologico, Fondazione "Monzino" IRCCS, Institute of Cardiology, University of Milan, Italy

\begin{abstract}
$\checkmark$
Purpose: To report the use of a new self-expanding endograft for percutaneous treatment of iatrogenic subclavian artery perforations.

Case Reports: The subclavian artery of 2 patients was inadvertently cannulated during percutaneous attempts to implant a permanent pacemaker in one and catheterize the subclavian vein in the other. Because both patients had serious comorbidities, endovascular repair of the subclavian perforations was performed using the Hemobahn endograft, a nitinol stent covered internally with expanded polytetrafluoroethylene. The endoprostheses were successfully deployed via an ipsilateral brachial artery access. No signs of endograft occlusion, migration, deformation, or fracture have been observed during followup at 12 and 10 months, respectively, in these patients.

Conclusions: The Hemobahn stent-graft appears well suited to repairing subclavian artery injuries. Longer follow-up will determine if the design of this endograft will resist compression in this vascular location.
\end{abstract}

J Endovasc Ther 2001;8:417-421

Key words: subclavian vein, venous access complication, arterial injury, nitinol stent

The subclavian vein is readily accessible via a supra- or infraclavicular approach, so it is frequently used for central venous access, central venous pressure monitoring, and prolonged parenteral nutrition. ${ }^{1,2}$ Transcutaneous subclavian vein puncture and cannulation is also the technique of choice for permanent transvenous pacemaker implantation. ${ }^{3} \mathrm{We}$ describe 2 cases in which the subclavian artery (SA) was inadvertently cannulated during attempted percutaneous catheterizations of

Address for correspondence and reprints: Antonio L. Bartorelli, MD, Centro Cardiologico, Fondazione "Monzino" IRCCS, Via Parea 4, 20138 Milan, Italy. Fax: 39-02-58002398; E-mail: albartorelli@cardiologicomonzino.it the central venous circulation. The iatrogenic complication was successfully managed by endovascular treatment with a self-expanding stent-graft.

\section{CASE REPORTS}

A 74-year-old severely obese woman with a history of insulin-dependent diabetes and hypertension presented to another hospital with heart failure and Mobitz-II A-V block, alternating with complete A-V block. Several attempts to implant a permanent pacemaker failed, and a temporary pacemaker was positioned in the apex of the right ventricle through the right 
femoral vein. The patient was transferred to our institute, where physical examination on admission showed cardiac failure with hypotension $(90 / 60 \mathrm{~mm} \mathrm{Hg}$ ) and reduced arterial $\mathrm{pO}_{2}(78 \mathrm{~mm} \mathrm{Hg})$. Cardiomegaly was evident on the chest radiograph.

Permanent pacemaker implantation was planned, and a left percutaneous subclavian vein approach was attempted with an 18-G needle. Slow return of dark blood suggested that the vein had been entered, so a guidewire and sheath were introduced for placement of the pacing lead. However, radiography disclosed the lead within the posterior silhouette of the heart and a right bundle branch block was evident on electrocardiography, indicating that the lead had been introduced in the left subclavian artery and positioned in the left ventricle. After consultation with the cardiothoracic surgeon, the patient was deemed at high risk for surgical intervention, so an endovascular approach to seal the perforation was elected.

The patient was pretreated with 5000 units of heparin, $325 \mathrm{mg}$ of aspirin, and prophylactic antibiotics. A 7-F internal mammary artery guide catheter was placed at the ostium of the left SA via the right femoral artery. Angiography showed the pacemaker lead entering the left SA (Fig. 1A). A 30-MHz Ultracross 3.2$F$ intravascular ultrasound (IVUS) catheter (CVIS, Boston Scientific/SCIMED, Natick, MA USA) was advanced over a 0.014-inch guidewire distal to the pacing lead insertion site. IVUS imaging (Fig. 1B) with automatic pullback confirmed the intraluminal position of the lead; measurements of the vessel diameters proximal and distal to the lead entry were recorded for stent-graft sizing.

To repair the injury, an 8-mm-wide, $10-\mathrm{cm}$ long Hemobahn endograft (Figs. 1C,D) (W.L. Gore \& Associates, Flagstaff, AZ, USA) was advanced over a 0.020-inch guidewire through a 9-F introducer inserted into the surgically exposed left brachial artery; the device was positioned under fluoroscopic control in the left SA and across the pacing lead entry site. The lead was then withdrawn, and the stent-graft was simultaneously deployed. An 8- $\times 20-\mathrm{mm}$ peripheral balloon catheter (PowerFlex Plus, Cordis, Roden, The Netherlands) inflated to 8 atmospheres was used to fully expand the stent-graft and optimize its apposition to the arterial wall.

Completion angiography (Fig. 1E) and IVUS imaging documented a satisfactory result without contrast extravasation. The introducer sheath was removed, and the brachial artery was repaired under local anesthesia. The next day, permanent pacemaker implantation was successfully performed through a surgical left cephalic vein approach. Endograft patency was demonstrated at 12 months by duplex ultrasonography; radiography of the left subclavian artery showed no displacement, deformation, or fracture of the stent-graft.

In the second case, an 83-year-old man suffered a stroke while recovering from elective coronary bypass surgery. Due to limited venous access, central venous catheterization was necessary for patient monitoring and infusion therapy. An attempt to cannulate the right subclavian vein from the infraclavicular approach resulted in placement of a 7-F, triple-lumen polyurethane CVP catheter in the SA (Fig. 2A). Owing to the critical condition of the patient, an endografting procedure was planned to repair the SA. Twenty-four hours later, an 8-mm-wide, 10-cm-long Hemobahn stent-graft was placed into the right SA via the surgically exposed right brachial artery using the technique described above. Postinterventional angiography (Fig. 2B) and IVUS imaging confirmed a satisfactory result. Normal arterial flow through the graft was documented by duplex scanning at the 10-month follow-up examination. No migration, deformation, or stent-graft fracture was seen on fluoroscopic examination of the right SA.

\section{DISCUSSION}

Accidental puncture of the SA is a recognized complication of percutaneous central venous catheter placement via the subclavian route. ${ }^{1,2}$ The mishap is usually easily recognized by the pulsating bright red blood return in the aspirating syringe. Usually no ill effect will result if a small gauge needle is used and promptly withdrawn without sideward motion. However, the accident is aggravated if arterial puncture is not recognized and an introducer is inserted. Catastrophic bleeding can result when the sheath is withdrawn. ${ }^{2}$ 

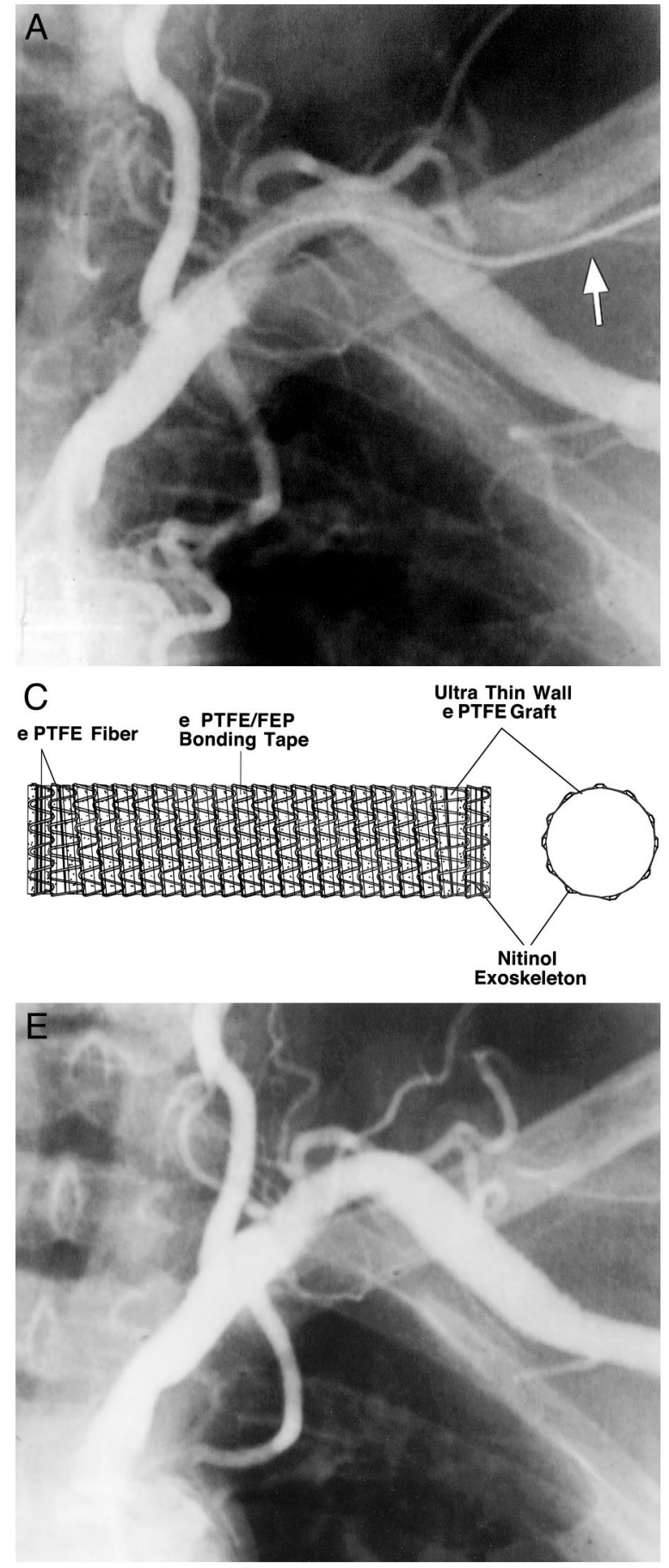

As the cases described here illustrate, inadvertent SA cannulation is often due to predisposing factors. Arterial entry may be entirely unrecognized because arterial desaturation makes the blood appear venous, or because hypotension or diminished arterial pulse pressure dampens pulsatile flow. Cen-
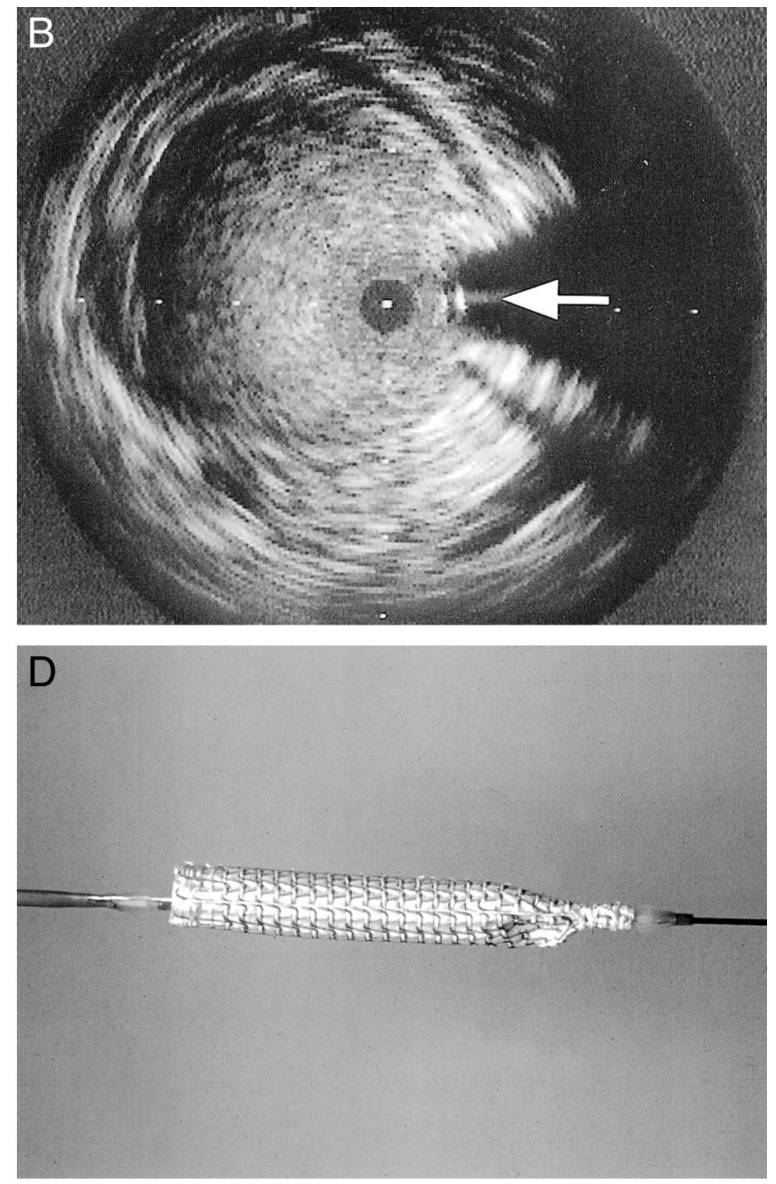

Figure $1 \diamond$ In the first patient, the preoperative angiogram (A) and the IVUS scan (B) show the pacemaker lead (arrow) penetrating the left subclavian artery. A Hemobahn stent-graft (C) consists of a selfexpanding nitinol exoskeleton lined internally by ultrathin expanded ePTFE that is bonded to the stent by a nonporous 1-mm-wide tape along its entire length. (D) The self-expanding Hemobahn is deployed by pulling a fiber that releases the knots along the endoprosthesis. (E) The completion arteriogram shows the wellpositioned endograft with no evidence of contrast extravasation or side branch obstruction.

tral venous access with Doppler ultrasoundguided needles has been proposed as an alternative to blind puncture, ${ }^{4}$ which may be particularly advantageous for patients in whom difficult venous cannulation is anticipated.

When the subclavian artery has been inad- 

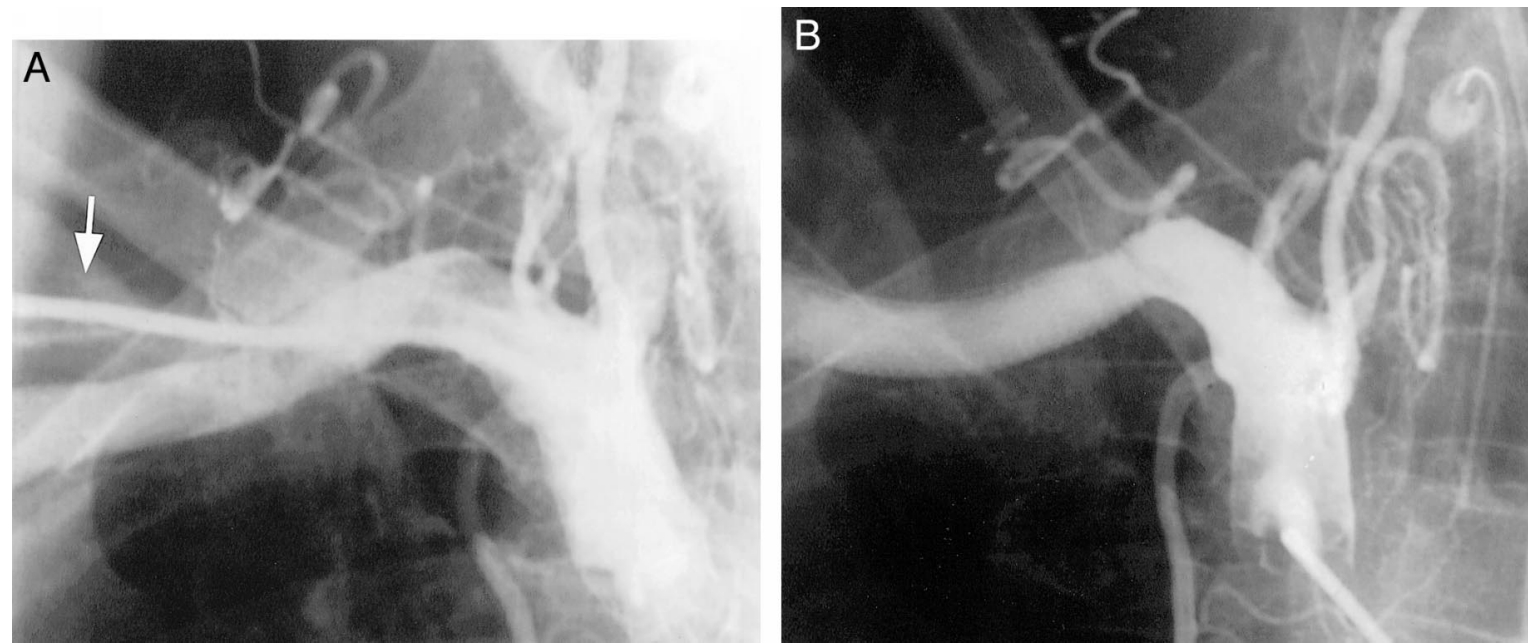

Figure $2 \triangleleft$ Preoperative angiogram (A) in the second patient showing a triple-lumen polyurethane CVP catheter (arrow) entering the right subclavian artery. After Hemobahn deployment, the completion angiogram (B) documents arterial patency and precise endograft positioning without endoleak or compromised subclavian branches.

vertently cannulated with large-caliber catheters, their immediate removal and application of pressure at the entry site may not prevent hematoma and potentially fatal hemorrhage. Therefore, surgical closure of the perforation under general anesthesia has been advocated, but surgical access is difficult in this location, and the potential for exsanguinating hemorrhage is ever present. ${ }^{5}$ Although supraand infraclavicular surgical exposures can be used, a more aggressive operative approach with median sternotomy, clavicular resection, or thoracotomy may be needed to obtain wider SA exposure and vascular control. ${ }^{6}$

Our patients were considered to be at elevated surgical and anesthetic risk because of their underlying medical conditions, so we decided to manage the complications with endovascular stent-grafts. Alternatively, we could have used a vascular closure device to seal the entry sites with the advantages of an easier procedure, lower cost, and avoidance of permanent implants. However, closure devices have a less than $100 \%$ success rate, and external manual compression is often required to achieve complete hemostasis, especially after collagen plug deployment. This would have been difficult and ineffective given the intrathoracic location of the injury. ${ }^{7}$ Even percutaneous suture-mediated devices require guidewire placement, which would have been difficult in the presence of a pacemaker lead. ${ }^{8}$

Endovascular repair of penetrating SA injuries and hemorrhage is well documented,, 10 but so too are the cases of graft compression and fracture in subclavian endografts. ${ }^{9,11,12}$ Initially, this complication was seen in devices made with easily deformable, longitudinally inflexible Palmaz stents, ${ }^{9}$ but self-expanding stent-grafts have also failed in this location. ${ }^{11,12}$ The Hemobahn endoprosthesis, which features a self-expanding nitinol stent covered internally with ultrathin expanded polytetrafluoroethylene, has been specifically designedfj for percutaneous endovascular treatment of peripheral arteries. ${ }^{13,14}$ The exoskeleton's longitudinal flexibility and shape memory should make this endograft resistant to deformation by mechanical forces and vascular flexure. In our 2 cases, the stent-grafts have remained widely patent for 10 and 12 months. However, more extensive, long-term clinical experience is needed to confirm the durability of the $\mathrm{He}$ mobahn device at flexion or extrinsic compression points.

Stent-graft failure in follow-up may be influenced by other factors, such as thrombogenicity and tissue response. Incomplete endothelialization of the graft may cause thrombosis, while exuberant neointimal hyperplasia may compromise patency. Experi- 
mental studies have demonstrated that the microstructure of the Hemobahn graft material promotes rapid endothelialization of flow surfaces, reducing the risk of thrombosis. ${ }^{15}$ However, there is no evidence that endothelialization of ePTFE occurs in human vessels, and clinical studies will be needed to confirm these promising results.

Because the Hemobahn stent-graft automatically expands to a predetermined diameter when deployed, an important technical component of successful implantation is careful assessment of vessel diameter to select a device of appropriate size. This can be performed by angiography or, more accurately, with IVUS imaging, which is also useful to evaluate correct graft deployment. Usually, an endoprosthesis with an unconstrained diameter $\sim 10 \%$ greater than the target vessel diameter is sufficient to allow secure fixation along the vascular segment.

The results obtained in our cases suggest that endovascular repair may be successful in the management of life-threatening subclavian artery perforations complicating central venous access, particularly in critically ill patients. However, longer follow-up will be necessary to fully evaluate the safety and efficacy of the Hemobahn device for this particular application.

\section{REFERENCES}

1. Venus B, Satish P. Vascular cannulation. In: Civetta JM, Taylor RW, Kirby RR, eds. Critical Care. 3rd ed. Philadelphia: Lippincott-Raven; 1997: 521-544.

2. Reich DL, Kaplan JA. Hemodynamic monitoring. In: Kaplan JA, ed. Cardiac Anesthesia. 3rd ed. Philadelphia: W.B. Saunders Company; 1993:261-298.

3. Hayes DL, Holmes DR, Furman S. Permanent pacemaker implantation. In: Furman S, Hayes DL, Holmes DR, eds. A Practice of Cardiac Pacing. 3rd ed. Armonk, NY: Futura Publishing Company; 1993:261-307.

4. Vucevic M, Tehan B, Gamlin F, et al. The
SMART needle. A new Doppler ultrasoundguided vascular access needle. Anaesthesia. 1994;49:889-891.

5. Brown $\mathrm{CQ}$. Inadvertent prolonged cannulation of the carotid artery. Anesth Analg. 1982;61: 150-152.

6. Perry MO. Vascular injuries in the neck and thoracic outlet. In: Wilson SE, Veith FJ, Hobson RW, et al, eds. Vascular Surgery: Principles and Practices. New York: McGraw-Hill, Inc.; 1987: 834-842.

7. Sanborn TA, Gibbs HH, Brinker JA, et al. A multicenter trial comparing a percutaneous collagen hemostasis device with conventional manual compression after diagnostic angiography and angioplasty. J Am Coll Cardiol. 1993;22: 1273-1279.

8. Gerkens U, Cattelans N, Lampe EG, et al. Management of arterial puncture site after catheterization procedures: evaluating a suture-mediated device. Am J Cardiol. 1999;83:16581663.

9. Patel AV, Marin ML, Veith FJ, et al. Endovascular graft repair of penetrating subclavian artery injuries. J Endovasc Surg. 1996;3:382-388.

10. Becker GJ, Benenati JF, Zemel G, et al. Percutaneous placement of a balloon-expandable intraluminal graft for life-threatening subclavian arterial hemorrhage. J Vasc Interv Radiol. 1991; 2:225-229.

11. Phipp LH, Scott DJA, Kessel D, et al. Subclavian stents and stent-grafts: cause for concern? $J$ Endovasc Surg. 1999;6:223-226.

12. Sitsen ME, Ho GH, Blankensteijn JD. Deformation of self-expanding stent-grafts complicating endovascular repair of peripheral aneurysms. J Endovasc Surg. 1999;6:288-292.

13. Bürger T, Meyer F, Tautenhahn J, et al. Initial experiences with percutaneous endovascular repair of popliteal artery lesions using a new PTFE stent-graft. J Endovasc Surg. 1998;5:365372.

14. Bartorelli AL, Trabattoni D. Endovascular treatment of two pseudoaneurysms of the superficial femoral artery with the Hemobahn graftstent. Int J Cardiovasc Interv. 1999;2:129.

15. Virmani R, Kolodgie FD, Dake MD, et al. Histopathologic evaluation of an expanded polytetrafluoroethylene-nitinol stent endoprosthesis in canine iliofemoral arteries. J Vasc Interv Radiol. 1999;10:445-456. 